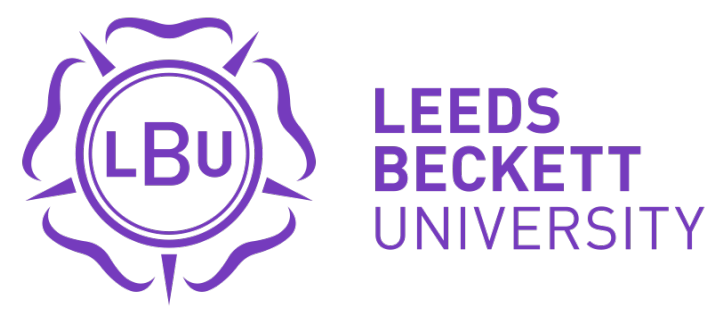

Citation:

Vajpayee, V and Becerra, V and Bausch, N and Banerjee, S and Deng, J and Shimjith, SR and Arul, AJ (2020) Robust Subspace Predictive Control based on Integral Sliding Mode for a Pressurized Water Reactor. 2020 7th International Conference on Control, Decision and Information Technologies (CoDIT). pp. 7-12. ISSN 2576-3555 DOI: https://doi.org/10.1109/CoDIT49905.2020.9263955

Link to Leeds Beckett Repository record:

https://eprints.leedsbeckett.ac.uk/id/eprint/6984/

Document Version:

Article (Accepted Version)

(C) 2020 IEEE. Personal use of this material is permitted. Permission from IEEE must be obtained for all other uses, in any current or future media, including reprinting/republishing this material for advertising or promotional purposes, creating new collective works, for resale or redistribution to servers or lists, or reuse of any copyrighted component of this work in other works.

The aim of the Leeds Beckett Repository is to provide open access to our research, as required by funder policies and permitted by publishers and copyright law.

The Leeds Beckett repository holds a wide range of publications, each of which has been checked for copyright and the relevant embargo period has been applied by the Research Services team.

We operate on a standard take-down policy. If you are the author or publisher of an output and you would like it removed from the repository, please contact us and we will investigate on a case-by-case basis.

Each thesis in the repository has been cleared where necessary by the author for third party copyright. If you would like a thesis to be removed from the repository or believe there is an issue with copyright, please contact us on openaccess@leedsbeckett.ac.uk and we will investigate on a case-by-case basis. 


\title{
Robust Subspace Predictive Control based on Integral Sliding Mode for a Pressurized Water Reactor
}

\author{
Vineet Vajpayee, Victor Becerra, Nils Bausch, Shohan Banerjee, Jiamei Deng, S. R. Shimjith, A. John Arul
}

\begin{abstract}
This work combines the subspace predictive control technique with the integral sliding mode control strategy to formulate a novel robust subspace predictive control scheme. The subspace predictive controller provides the nominal control whereas the integral sliding mode controller gives the discontinuous control action. The aim is to improve the capability of subspace predictive controller in handling uncertainties and external disturbances. The proposed control scheme is evaluated with a simulated pressurized water nuclear reactor. The effectiveness of the proposed technique is demonstrated for two different load-following operations in the presence of uncertainties.
\end{abstract}

\section{INTRODUCTION}

Model predictive control (MPC) is extensively employed in the process industries because of its capability to handle constraints, adaptability to new operating conditions, and its optimal performance. Predictive controllers solve an optimization problem at each sampling instant to calculate future control input over a time period [1]. The effective implementation of a MPC requires an accurate mathematical model of the underlying process. Since a nuclear power plant is a highly-constrained complex non-linear system which is usually subject to parameter variations due to fuel burnup, internal reactivity feedbacks, and other uncertainties, the traditional MPC design techniques yield sub-optimal performance [2]. Moreover, the requirement of an accurate model is quite stringent especially when retrofitting new controllers in an aged nuclear power plant. These conditions may further increase the complexity of the controller due to conservative nature of constraints. Thus, the control algorithm in a nuclear power plant should be designed in such a way that it tackles these problems and provides a near-optimal control performance without increasing the computational complexity significantly.

Subspace predictive control (SPC) is a recently developed predictive control strategy based on subspace matrix structure [3]-[6]. It combines the estimation of linear predictor using

Vineet Vajpayee (vineet.vajpayee@port.ac.uk), Victor Becerra (victor.becerra@port.ac.uk), and Nils Bausch (nils.bausch@port.ac.uk) are with School of Energy and Electronic Engineering, University of Portsmouth, Portsmouth, PO1 3DJ, United Kingdom.

Shohan Banerjee (s.banerjee@leedsbeckett.ac.uk) and Jiamei Deng (j.deng@leedsbeckett.ac.uk) are with School of Built Environment, Engineering, and Computing, Leeds Beckett University, Leeds, LS6 3QS, United Kingdom.

S. R. Shimjith (srshim@barc.gov.in) is with Reactor Control System Design Section, Bhabha Atomic Research Centre, Mumbai, 400 085, India and Homi Bhabha National Institute, Mumbai, 400 094, India.

A. John Arul (arul@igcar.gov.in) is with Probabilistic Safety, Reactor Shielding and Nuclear Data Section, Indira Gandhi Centre for Atomic Research, Kalpakkam, 603 102, India. subspace identification with the formulation of receding horizon control design. In contrast to the traditional MPC, which first models the process and then estimates the controller parameters, the SPC combines these two steps into one, thereby reducing the computation time, complexity, and errors arising due to model-plant mismatch. SPC directly designs the controller from the recorded measurement data. The formulation of SPC neither requires the solution of a nonlinear Riccati equation, as is the case with linear quadratic control, nor the solution of a recursive Diophantine equation, as with generalised predictive control. The realization of SPC is through singular value and $\mathrm{QR}$ decompositions, which make the algorithm numerically stable and computationally efficient.

Although predictive controller design approaches optimize the performance of constrained systems, they lack uncertainty or disturbance handling capabilities. Over the last few decades, different robust predictive controller design techniques have been suggested to resolve these problems. For instance, min-max approach [7], open loop nominal approach [8], constraint tightening approach [9], etc. However, these methods have limited applicability in case of large complex systems such as a nuclear power plant as they are effective for systems with few states or systems with slow dynamics [10]. Moreover, these methods carry a very high computational burden [11].

In another approach, a predictive controller is fused with sliding mode control (SMC) to form a new hybrid control strategy. SMC is a well-known robust control design technique which guarantees robustness towards matched uncertainties, that is, uncertainties entering through the input channel, once the system reaches the sliding surface [12]. The idea of SMC is to devise a discontinuous control action which steers the system towards a stable sliding surface and then maintains the system on this surface [13]. SMC possesses very low computational complexity and assures finite time convergence to the sliding surface. In the literature, these techniques have been combined to incorporate the complementary strengths of predictive control and SMC in two different ways. In the first strategy, referred to as predictive sliding mode control, the predictive controllers are employed to update sliding surface parameters [14]-[16]. It is indicated that incorporation of a predictive controller improves the performance during reaching phase. A different way of integrating predictive control with SMC is proposed by [17]-[19]. In these techniques the control action consists of two components, the first component is the nominal control which is generated by the predictive control technique 
and the second component is the discontinuous control which is generated by the integral sliding mode control (ISMC). One of the main advantages of ISMC over traditional SMC is that the former avoids the reaching phase by enforcing the system trajectories to lie on the sliding surface from the very beginning [13], [20]. Nevertheless, the above mentioned approaches rely on the accuracy of the nominal model of the system.

In this work, a robust subspace predictive control (RSPC) approach is proposed by integrating SPC with ISMC to enhance robustness and reduce the computational burden. The proposed control scheme is able to overcome the uncertainties and disturbances present in the system without affecting the nominal system performance. The efficacy of the proposed RSPC is validated during two different loadfollowing transients of a pressurized water reactor (PWR). The control performance of the proposed RSPC is further compared with that of the classical SPC.

The rest of the paper is organized as follows: Section II formulates the problem. Section III presents the proposed control scheme. Section IV evaluates the proposed technique on a simulated PWR-type nuclear reactor and discusses its effectiveness through simulation results. Conclusions are drawn in section $\mathrm{V}$ indicating main contributions.

\section{Problem Formulation}

Let us consider a linear uncertain system, represented in state-space form as

$$
\begin{aligned}
\dot{x}(t) & =A x(t)+B(u(t)+\xi(t)), \\
y(t) & =C x(t),
\end{aligned}
$$

where $x(t) \in \mathbb{R}^{n}, u(t) \in \mathbb{R}^{m}, y(t) \in \mathbb{R}^{l}$, and $\xi(t) \in$ $\mathbb{R}^{m}$ respectively represent state vector, control input, system output, and matched uncertainty. $A, B$, and $C$ are system matrices. It is assumed that $(A, B)$ is controllable and that the system uncertainties are unknown and bounded, so that

$$
\|\xi(t)\| \leq \xi^{*}, \quad \xi^{*}>0
$$

The control aim is to force the system output $y(t)$ to follow the desired reference $r(t)$ with minimal control effort in the presence of uncertainty $\xi(t)$. To achieve this objective the control scheme, depicted in Fig. 1, is proposed in this work, where the ISMC reduces the effect of uncertainties and the SPC guarantees minimum control effort.

\section{Proposed Control Approach}

For the proposed control strategy, the control law $u(t)$, according to Fig. 1, is formed of two parts, i.e.,

$$
u(t)=u_{n}(t)+u_{d}(t)
$$

where the nominal control $\left(u_{n}(t)\right)$ is produced using SPC to obtain nominal system performance in an optimal way whereas the discontinuous control $\left(u_{d}(t)\right)$ is generated by ISMC to compensate for uncertainties. Thus, (1) can be

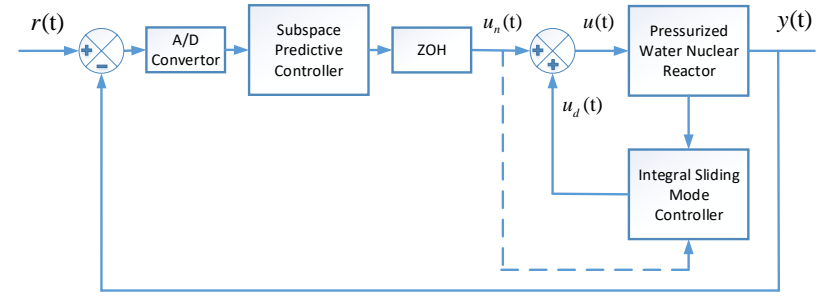

Fig. 1. Block diagram of the proposed RSPC algorithm.

written as

$$
\begin{aligned}
\dot{x}(t) & =A x(t)+B\left(u_{n}(t)+u_{d}(t)+\xi(t)\right), \\
y(t) & =C x(t) .
\end{aligned}
$$

\section{A. Design of Nominal Control by SPC}

The nominal system is given by

$$
\begin{aligned}
& \dot{x}_{n}(t)=A x_{n}(t)+B u_{n}(t)+K e(t), \\
& y_{n}(t)=C x_{n}(t)+e(t),
\end{aligned}
$$

where $x_{n}(t)$ represent the system states due to nominal control. The objective of SPC is to find $u_{n}(t)$ by minimizing the following cost function,

$$
J=\left(\hat{\mathbf{y}}_{\mathbf{f}}-\mathbf{r}_{\mathbf{f}}\right)^{T} Q_{f}\left(\hat{\mathbf{y}}_{\mathbf{f}}-\mathbf{r}_{\mathbf{f}}\right)+\Delta \mathbf{u}_{\mathbf{f}}^{T} R_{f} \Delta \mathbf{u}_{\mathbf{f}},
$$

where

$$
\begin{aligned}
& \hat{\mathbf{y}}_{\mathbf{f}}=\left[\begin{array}{llll}
\hat{y}^{T}[t+1] & \hat{y}^{T}[t+2] & \cdots & \hat{y}^{T}\left[t+N_{p}\right]
\end{array}\right]_{T}^{T}, \\
& \mathbf{r}_{\mathbf{f}}=\left[\begin{array}{llll}
r^{T}[t+1] & r^{T}[t+2] & \cdots & r^{T}\left[t+N_{p}\right]
\end{array}\right]^{T}, \\
& \mathbf{u}_{\mathbf{f}}=\left[\begin{array}{llll}
u_{n}^{T}[t+1] & u_{n}^{T}[t+2] & \cdots & u_{n}^{T}\left[t+N_{c}\right]
\end{array}\right]^{T} \\
& \text { respectively represent predicted output, desired reference, }
\end{aligned}
$$
and future input sequence. The difference operator is $\Delta=1-z^{-1}$, where $z^{-1}$ is backward shift operator. $N_{c}\left(\leq N_{p}\right)$ and $N_{p}$ are control and prediction horizons respectively. $R_{f}=I_{N_{c}} \otimes R$ penalizes the rate of change of input, where $R \in \mathbb{R}^{m \times m}$ is a positive definite matrix, $I_{N_{c}}$ is an $N_{c} \times N_{c}$ identity matrix, and $\otimes$ denotes the Kronecker product. Similarly, $Q_{f}=I_{N_{p}} \otimes Q$ penalizes the error between desired reference and output, where $Q \in \mathbb{R}^{l \times l}$ is a positive semi-definite matrix. The formulation of SPC requires the design of a predictor to provide the control law. This is discussed as follows:

The block Hankel matrices can be formed from the collected measurement data as

$$
\begin{aligned}
Y_{P}= & {\left[\begin{array}{cccc}
y[1] & y[2] & \cdots & y[N-2 f+1] \\
y[2] & y[3] & \cdots & y[N-2 f+2] \\
\vdots & \vdots & \ddots & \vdots \\
y[f] & y[f+1] & \cdots & y[N-f]
\end{array}\right] ; \quad \text { (7) } } \\
Y_{F}= & {\left[\begin{array}{cccc}
y[f+1] & u[f+2] & \cdots & y[N-f+1] \\
y[f+2] & u[f+3] & \cdots & y[N-f+2] \\
\vdots & \vdots & \ddots & \vdots \\
y[2 f] & u[2 f+1] & \cdots & f[N]
\end{array}\right] \text { (8) } }
\end{aligned}
$$

where $f$ is the order of predictor matrix. $Y_{P} \in$ 
$\mathbb{R}^{f l \times(N-2 f+1)}$ and $Y_{F} \in \mathbb{R}^{f l \times(N-2 f+1)}$ are called past and future output data Hankel matrices respectively. In a similar fashion, $U_{P} \in \mathbb{R}^{f m \times(N-2 f+1)}$ and $X_{P} \in \mathbb{R}^{n \times(N-2 f+1)}$ are defined as past input and past state matrices respectively. The same notation holds with subscript $F$ terms to define future Hankel matrices. Using these definition one can write,

$$
\begin{aligned}
& Y_{P}=\Gamma_{f} X_{P}+H_{f}^{d} U_{P}+H_{f}^{s} E_{P}, \\
& Y_{F}=\Gamma_{f} X_{F}+H_{f}^{d} U_{F}+H_{f}^{s} E_{F}, \\
& X_{F}=A^{f} X_{P}+\Delta_{f}^{d} U_{P}+\Delta_{f}^{s} E_{P},
\end{aligned}
$$

where $\Gamma_{f} \in \mathbb{R}^{f l \times n}$ is the extended observability matrix. $H_{f}^{d} \in \mathbb{R}^{f l \times f m}$ and $H_{f}^{s} \in \mathbb{R}^{f l \times f l}$ are deterministic and stochastic lower block-triangular Toeplitz matrix. $\Delta_{f}^{d} \in$ $\mathbb{R}^{n \times f m}$ and $\Delta_{f}^{s} \in \mathbb{R}^{n \times f l}$ are deterministic and stochastic reverse extended controllability matrix. Thus, from (10), the predictor is given by

$$
\hat{Y}_{F}=L_{w} W_{P}+L_{u} U_{F},
$$

where $W_{P}=\left[\begin{array}{cc}Y_{P}^{T} & U_{P}^{T}\end{array}\right]^{T} \in \mathbb{R}^{f(m+l) \times(N-2 f+1)} . L_{w} \in$ $\mathbb{R}^{f l \times f(m+l)}$ and $L_{u} \in \mathbb{R}^{f l \times f m}$ are predictor matrices.

Now, in order to incorporate the above-defined predictor in SPC, it is sufficient to consider only the leftmost column of $\hat{Y}_{f}$. Thus, (10) can be rewritten as

$$
\hat{\mathbf{y}}_{\mathbf{f}}=L_{w} \mathbf{w}_{\mathbf{p}}+L_{u} \mathbf{u}_{\mathbf{f}},
$$

or simply in terms of input increments as,

$$
\hat{\mathbf{y}}_{\mathbf{f}}=\overline{\mathrm{I}}_{l} y[t]+O_{l} L_{w} \Delta \mathbf{w}_{\mathbf{p}}+O_{l} L_{u} \Delta \mathbf{u}_{\mathbf{f}},
$$

where

$O_{l}=\left[\begin{array}{cccc}I_{l} & 0 & \cdots & 0 \\ I_{l} & I_{l} & \cdots & 0 \\ \vdots & \vdots & \ddots & \vdots \\ I_{l} & I_{l} & \cdots & I_{l}\end{array}\right] \in R^{N_{p} l \times N_{p} l}, \overline{\mathrm{I}}_{l}=\left[\begin{array}{c}I_{l} \\ I_{l} \\ \vdots \\ I_{l}\end{array}\right] \in R^{N_{p} l \times l}$

and $I_{l}$ is an $l \times l$ identity matrix. Rewriting (12), using $\overline{\mathbf{y}}[t]=$ $\overline{\mathrm{I}}_{l} y[t], \bar{L}_{w}=O_{l} L_{w}$, and $\bar{L}_{u}=O_{l} L_{u}$, as

$$
\hat{\mathbf{y}}_{\mathbf{f}}=\overline{\mathbf{y}}[t]+\bar{L}_{w} \Delta \mathbf{w}_{\mathbf{p}}+\bar{L}_{u} \Delta \mathbf{u}_{\mathbf{f}}
$$

Thus, the input increment is computed by

$\Delta \mathbf{u}_{\mathbf{f}}=-\left(\left(\bar{L}_{u}\right)^{T} Q_{f} \bar{L}_{u}+R_{f}\right)^{-1}\left(\bar{L}_{u}\right)^{T} Q_{f}\left(\overline{\mathbf{y}}[t]-\mathbf{r}_{\mathbf{f}}+\bar{L}_{w} \Delta \mathbf{w}_{\mathbf{p}}\right)$,

or simply,

$$
\Delta \mathbf{u}_{\mathbf{f}}=-K_{u}\left(\overline{\mathbf{y}}[t]-\mathbf{r}_{\mathbf{f}}\right)-K_{w} \Delta \mathbf{w}_{\mathbf{p}}
$$

where the gain matrices $\left(K_{u}\right.$ and $\left.K_{w}\right)$ are defined as

$$
\begin{aligned}
& K_{u}=\left(\left(\bar{L}_{u}\right)^{T} Q_{f} \bar{L}_{u}+R_{f}\right)^{-1}\left(\bar{L}_{u}\right)^{T} Q_{f}, \\
& K_{w}=\left(\left(\bar{L}_{u}\right)^{T} Q_{f} \bar{L}_{u}+R_{f}\right)^{-1}\left(\bar{L}_{u}\right)^{T} Q_{f} \bar{L}_{w} .
\end{aligned}
$$

Finally, the nominal control signal is updated using only the first element of the nominal control move

$$
u_{n}(t+1)=\Delta \mathbf{u}_{\mathbf{f}}(1)+u_{n}(t) .
$$

\section{B. Design of Discontinuous Control by ISMC}

The ISMC works by designing first an integral sliding surface followed by the design of a discontinuous control law. The sliding motion occurs on the integral sliding surface whereas the control law drives the system trajectories onto the sliding surface and maintained on it. To preserve the nominal closed loop system performance, an integral sliding surface $\phi(t) \in \mathbb{R}^{m}$ can be designed as [13]

$$
\phi(t)=B^{\dagger}\left[x(t)-x(0)-\int_{0}^{t} \dot{x}_{n}(\tau) d \tau\right],
$$

where $B^{\dagger}=\left(B^{\top} B\right)^{-1} B^{\top}$ is left-pseudo inverse of input distribution matrix $B$. The term $-x(0)$ assures that the system starts from the sliding surface by eliminating the reaching phase and enforcing $\phi(0)=0$. Thus, the closedloop system turns out to be robust towards matched uncertainties from the initial time instant.

In this work, the discontinuous control $u_{d}(t)$ is formulated based on the reachability condition [12], [20]

$$
u_{d}(t)=-\mu \operatorname{sign}(\phi(t))
$$

where $\mu$ is an appropriately designed positive constant and $\operatorname{sign}($.$) is the standard signum function.$

\section{Stability Analysis}

1) SPC: In this section the asymptotic stability of the SPC is analysed. If $f \rightarrow \infty$, the gain matrices can be rewritten as

$$
\begin{aligned}
K_{u} & =\left(\left(\bar{H}_{f}^{d}\right)^{T} Q_{f} \bar{H}_{f}^{d}+R_{f}\right)^{-1}\left(\bar{H}_{f}^{d}\right)^{T} Q_{f}, \\
K_{w} & =\left(\left(\bar{H}_{f}^{d}\right)^{T} Q_{f} \bar{H}_{f}^{d}+R_{f}\right)^{-1}\left(\bar{H}_{f}^{d}\right)^{T} Q_{f} O_{l} \Gamma_{f} \hat{x}(t) .
\end{aligned}
$$

where $\bar{H}_{f}^{d}=O_{l} H_{f}^{d}$ and $\hat{x}(t)$ represent the state estimated by the Kalman filter,

$$
\hat{x}(q+1)=A x_{n}(q)+B u_{n}(q)+K\left(\mathrm{y}_{n}(\mathrm{q})-\mathrm{C} \hat{x}_{n}(q)\right)
$$

where $q=t-1, t-2, \cdots t-f$. Since $f \rightarrow \infty$, the Kalman filter is at steady state with steady state gain. Thus, the unconstrained SPC is asymptotically stable.

2) ISMC: In this subsection, the reachability of the integral sliding surface $\phi(t)$ is analysed. Choosing a Luapunov function $V(t)$ as

$$
V(t)=\frac{1}{2} \phi^{2}(t)
$$

Differentiating $V(t)$ with respect to time, we obtain

$$
\begin{aligned}
\dot{V}(t)= & \phi^{\top}(t) \dot{\phi}(t) \\
= & \phi^{\top}(t)\left(B^{\dagger} \dot{x}(t)-B^{\dagger} \dot{x}_{n}(t)\right) \\
= & \phi^{\top}(t)\left(B^{\dagger} A x(t)+u_{n}(t)+u_{d}(t)+\xi(t)\right. \\
& \left.-B^{\dagger} A x_{n}(t)-u_{n}(t)\right) \\
= & \phi^{\top}(t)\left(B^{\dagger} A\left(x(t)-x_{n}(t)\right)-\mu \operatorname{sign}(\phi(t))+\xi(t)\right) .
\end{aligned}
$$


During sliding mode the system trajectories follow the nominal system trajectories i.e., $x(t)=x_{n}(t)$. Thus, (24) becomes

$$
\begin{aligned}
\dot{V}(t) & =\phi^{\top}(t)(\xi(t)-\mu \operatorname{sign}(\phi(t))) \\
& =\phi^{\top}(t) \xi(t)-\mu \phi^{\top}(t) \operatorname{sign}(\phi(t)) \\
& \leq\|\phi(t)\|\|\xi(t)\|-\mu\|\phi(t)\| \\
& \leq\|\phi(t)\|\left(\xi^{*}-\mu\right) .
\end{aligned}
$$

Thus, for any choice of $\mu \geq \xi^{*}+\delta$, (25) becomes

$$
\dot{V}(t)=\phi^{\top}(t) \dot{\phi}(t) \leq-\delta\|\phi(t)\|,
$$

where $\delta$ is a small positive constant.

It is apparent from (26) that the trajectories of uncertain system (1) will be maintained on the sliding surface $\phi(t)=$ 0 and drive towards the specified equilibrium point despite the uncertainties in finite time. The boundary layer approach can be used to restraint the effect of chattering due to the presence of signum function in (20). The signum function can be approximated as,

$$
\operatorname{sign}(\phi(\mathrm{t}))=\frac{\phi(\mathrm{t})}{\|\phi(\mathrm{t})\|+\varepsilon}
$$

where $\varepsilon$ is a small positive constant.

\section{Case Study: Application to a Simulated PWR-TYPE NUCLEAR REACTOR}

For the purposes of identification and control, the dynamical model of a PWR can be suitably defined using the point kinetics equation with six groups of delayed neutrons precursors' concentration coupled with thermal hydraulics. The model is based on the following assumptions: The primary loop is characterized by a lumped model. The pressure and mass flow rate are constants. The heat generated in the core is transferred using single phase coolant. The primary loop model equations are as follows:

$$
\begin{aligned}
\frac{d P}{d t} & =\frac{\rho_{T}-\sum_{i=1}^{6} \beta_{i}}{\Lambda} P+\frac{\sum_{i=1}^{6} \beta_{i} C_{i}}{\Lambda}, \\
\frac{d C_{i}}{d t} & =\lambda_{i}\left(P-C_{i}\right), \quad i=1,2, \ldots, 6, \\
\frac{d T_{f}}{d t} & =H_{f} P-\gamma_{f}\left(T_{f}-T_{c}\right), \\
\frac{d T_{c}}{d t} & =-H_{c}\left(T_{\text {out }}-T_{\text {in }}\right)+\gamma_{c}\left(T_{f}-T_{c}\right), \\
T_{c} & =\frac{T_{\text {out }}+T_{\text {in }}}{2}, \\
\rho_{T} & =\rho+\alpha_{f} T_{f}+\alpha_{c} T_{c} .
\end{aligned}
$$

where $P$ is normalized neutronic power; $\beta_{i}, \lambda_{i}$, and $C_{i}$ denote fraction of delayed neutrons, decay constant, and normalized delayed neutron precursors' concentration of $i^{t h}$ group respectively; $\Lambda$ represents prompt neutron life time; $\rho$ and $\rho_{T}$ denote reactivity contributed by control input and the total reactivity respectively; $T_{f}$ and $T_{c}$ are temperatures of fuel and coolant respectively; $\alpha_{f}$ and $\alpha_{c}$ are temperature coefficients of reactivity of fuel and coolant respectively; $H_{f}$,
TABLE I

NEUTRONIC AND THERMAL-HYDRAULIC PARAMETERS

\begin{tabular}{|c|c|c|c|c|c|c|}
\hline Group, i & 1 & 2 & 3 & 4 & 5 & 6 \\
\hline$\lambda_{i}\left(s^{-1}\right)$ & 0.0125 & 0.0308 & 0.1152 & 0.3109 & 1.240 & 3.3287 \\
\hline$\beta_{i}$ & 0.000216 & 0.001416 & 0.001349 & 0.00218 & 0.00095 & 0.000322 \\
\hline \hline$H_{f}\left({ }^{\circ} \mathrm{Cs}^{-1}\right)$ & $H_{c}\left(s^{-1}\right)$ & $\gamma_{f}\left(s^{-1}\right)$ & $\gamma_{c}\left(s^{-1}\right)$ & $\alpha_{f}\left({ }^{\circ} \mathrm{C}^{-1}\right)$ & $\alpha_{c}\left({ }^{\circ} \mathrm{C}^{-1}\right)$ & $\Lambda(s)$ \\
102.7 & 0.2401 & 0.1792 & 0.0124 & $-2 \times 10^{-5}$ & $-5 \times 10^{-5}$ & $5 \times 10^{-4}$ \\
\hline$\tau_{\text {cold }}(s)$ & $\tau_{\text {hot }}(s)$ & $\tau_{s g}(s)$ & $D_{1}\left({ }^{\circ} \mathrm{Cs}^{-1}\right)$ & $D_{2}$ & \multicolumn{2}{|c|}{$D_{3}$} \\
7.0 & 5.0 & 11.3 & 3.746 & 0.7005 & \multicolumn{2}{c}{-0.2995} \\
\hline
\end{tabular}

$H_{c}, \gamma_{f}$, and $\gamma_{c}$ are proportionality constants; The dynamic equations governing the secondary side are given by

$$
\begin{aligned}
\frac{d T_{\text {in }}}{d t} & =\frac{1}{\tau_{\text {cold }}}\left(D_{2} T_{s g}-D_{3} T_{\text {hot }}-T_{\text {in }}\right), \\
\frac{d T_{h o t}}{d t} & =\frac{1}{\tau_{\text {hot }}}\left(T_{\text {out }}-T_{\text {hot }}\right) \\
\frac{d T_{s g}}{d t} & =-\frac{1}{\tau_{s g}}\left(T_{s g}-T_{\text {hot }}\right)-D_{1} L_{T},
\end{aligned}
$$

where $T_{\text {out }}, T_{i n}, T_{h o t}$, and $T_{s g}$ are temperatures of coreoutlet, core-inlet, hot leg and steam generator respectively. $\tau_{\text {cold }}, \tau_{\text {hot }}$, and $\tau_{s g}$ are time constants; $D_{1}, D_{2}$, and $D_{3}$ are constants; $L_{T}$ is turbine load. Values of various parameters used in (28)-(36) are listed in Table I [6].

It is assumed that initially the reactor is operating at $50 \%$ full power (FP). The system given by (28)-(36) is perturbed by a reactivity transient to obtain the corresponding variation in reactor power. The reactivity and power are considered as input and output of the reactor system respectively and thus form the estimation dataset for designing the nominal control. The values of nominal control design parameters are taken as $N_{P}=5, N_{C}=5, Q=0.1$, and $R=20$.

A case study is carried out to examine the robustness of the proposed controller in the presence of parametric uncertainties. A time varying parametric uncertainty in the form of $\xi(t)=0.01 \sin (0.05 \pi t)$ is assumed to be present during the load-following transient. Initially, the reference power is kept at $50 \% \mathrm{FP}$ for $36 \mathrm{~s}$; then it is varied at a rate of 5\%/min for $108 \mathrm{~s}$; kept at $59 \%$ FP for the next $108 \mathrm{~s}$; bring back to $50 \% \mathrm{FP}$ at the same rate and held at 50\% FP for 108 s; a 5\% step increment applied at $468^{\text {th }} \mathrm{s}$; kept at 55\% FP for $108 \mathrm{~s}$; a $5 \%$ step decrement applied at $576^{\text {th }} \mathrm{s}$ and kept at 50\% FP afterwards. The desired reference variation and the performance of the proposed RSPC controller and that of the classical SPC controller for tracking the reference are shown in Fig. 2. It can be observed that the proposed RSPC is able to track the variation smoothly as envisaged in spite of uncertainties, while the SPC fails in tracking the demand variation. Variations of control signal and rate of change of control signal are shown in Figs. 3 and 4, respectively. The variation of the sliding surface is shown in Fig. 5.

Another case study is performed to investigate the effect of unmodelled reactivity feedback due to xenon poisoning. Xenon concentration significantly affects a nuclear power plant behaviour because of very large thermal neutron absorption cross section. To study the effect of xenon build up, the simulations are carried out for a period of 16 hours. Initially, the reference power is kept at $50 \% \mathrm{FP}$ for $0.5 \mathrm{~h}$; then 


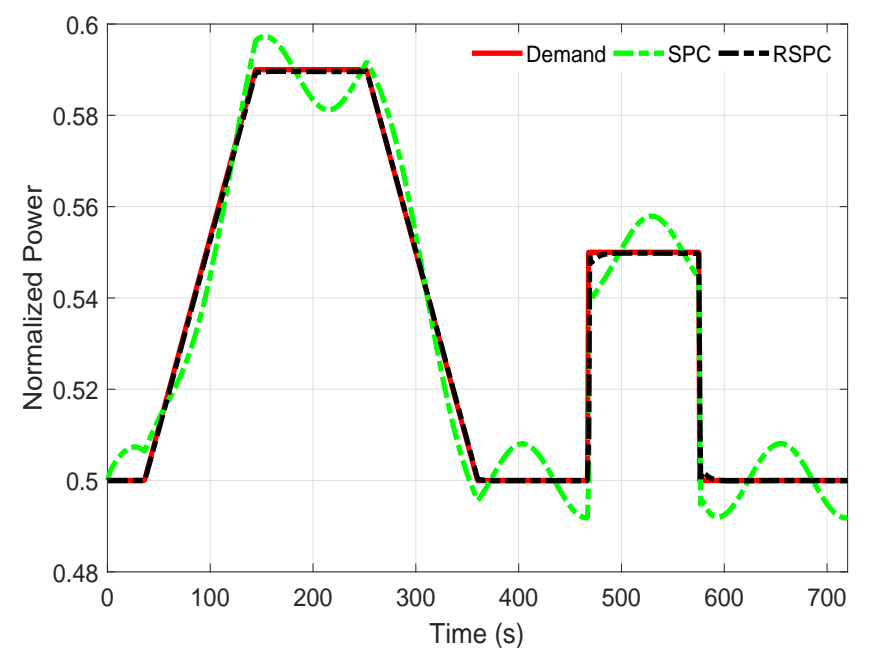

Fig. 2. Variation of reactor power for SPC and RSPC.

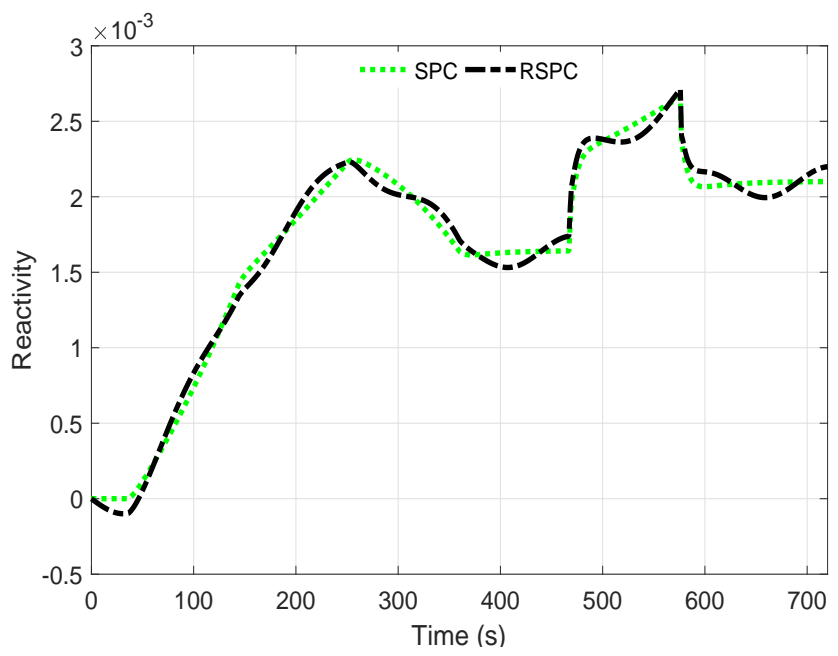

Fig. 3. Variation of control input for SPC and RSPC.

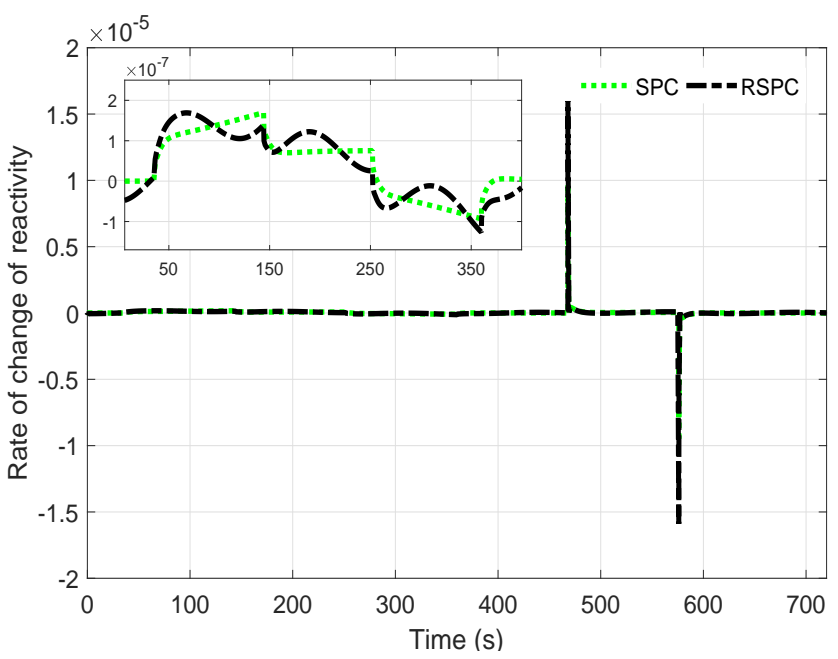

Fig. 4. Variation of rate of change of control input for SPC and RSPC.

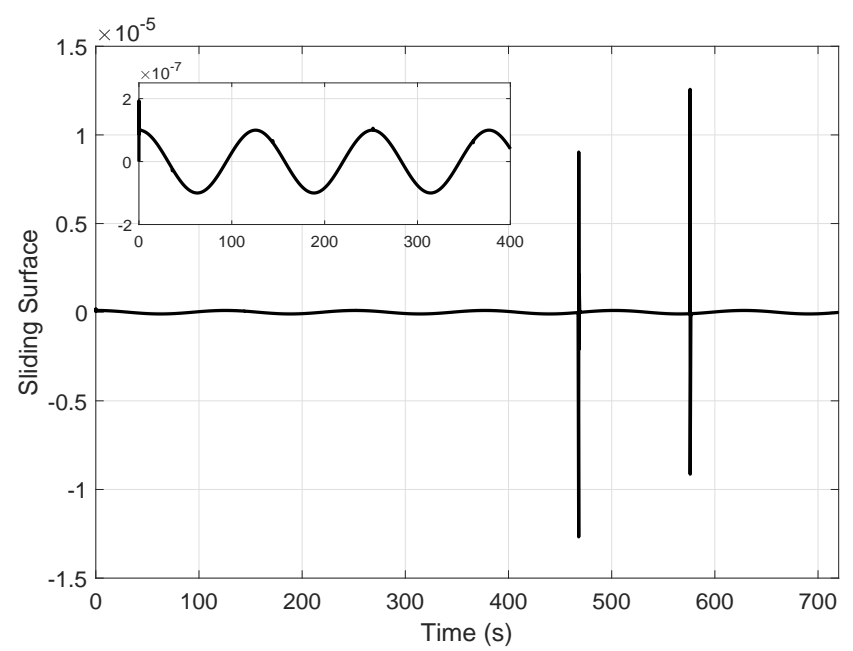

Fig. 5. Variation of sliding surface for RSPC.

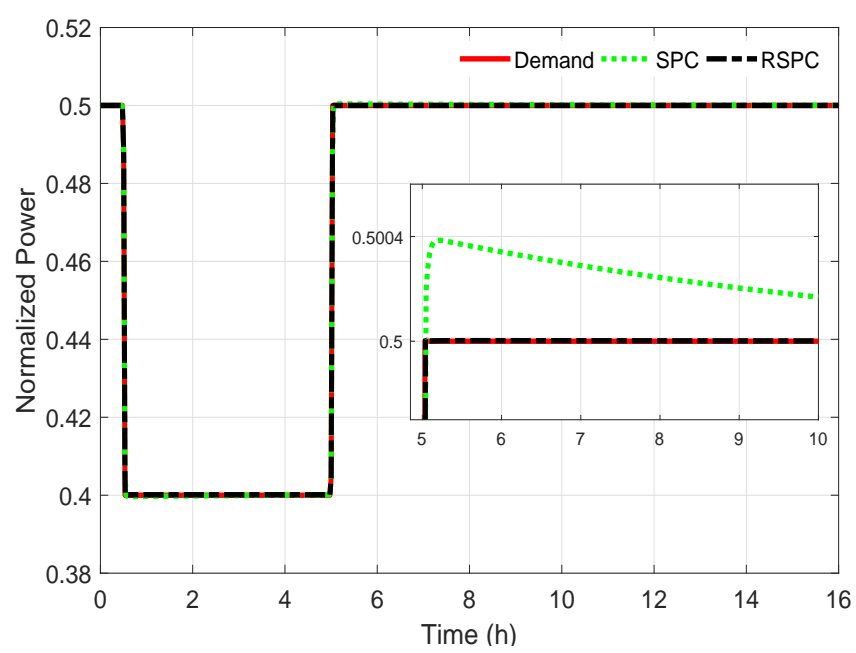

Fig. 6. Variation of reactor power for SPC and RSPC.

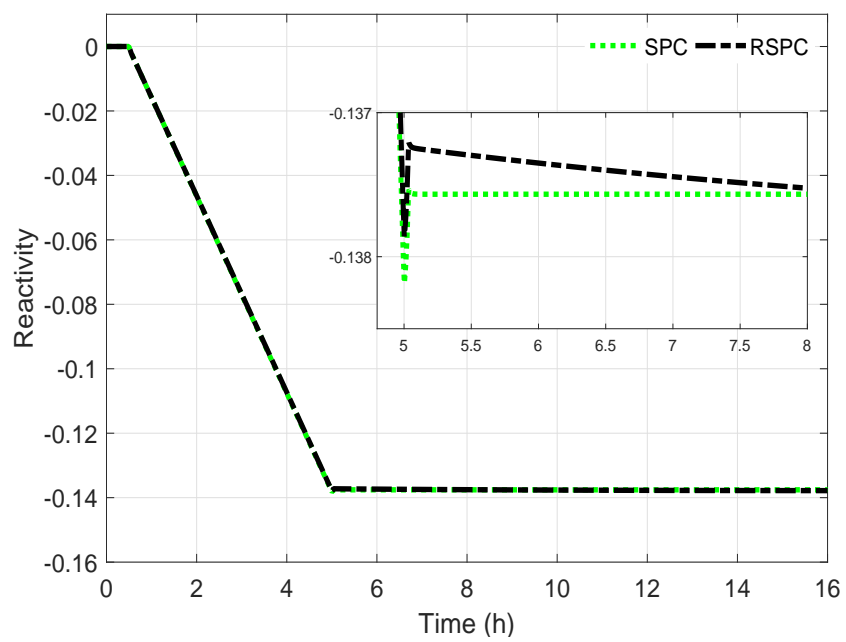

Fig. 7. Variation of control input for SPC and RSPC. 


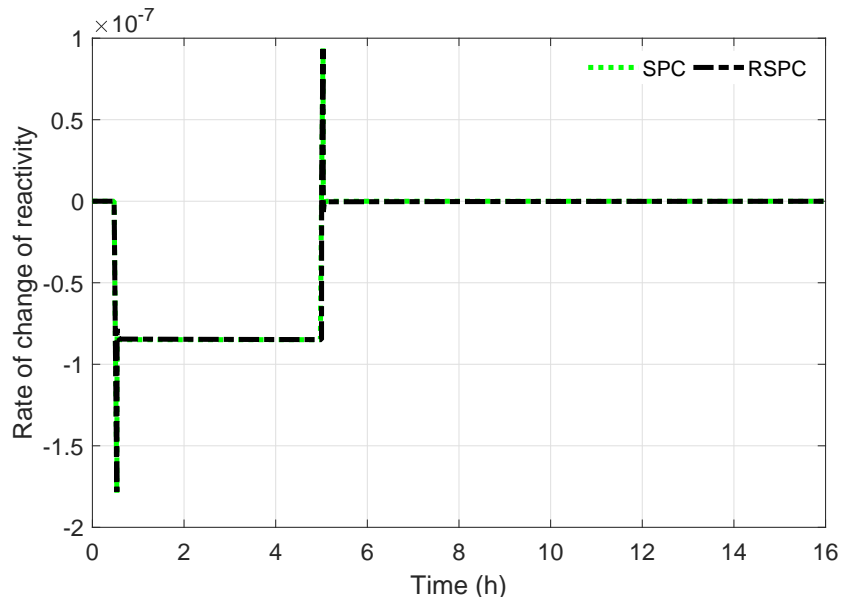

Fig. 8. Variation of rate of change of control input for SPC and RSPC.

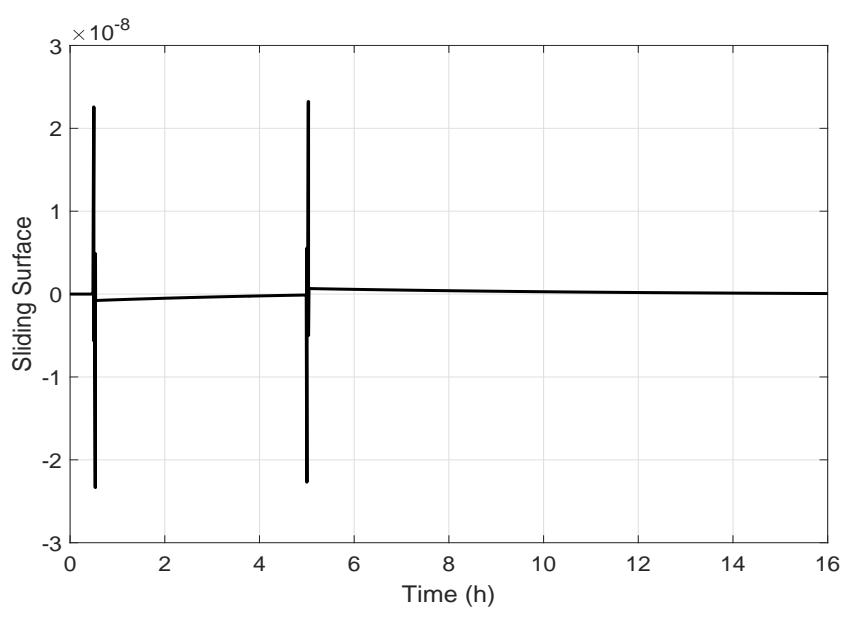

Fig. 9. Variation of sliding surface for robust SPC.

it is reduced from $50 \%$ to $40 \% \mathrm{FP}$ at a rate of $5 \% / \mathrm{min}$; kept at $40 \% \mathrm{FP}$ for the next $4.47 \mathrm{~h}$; then it is raised to $50 \% \mathrm{FP}$ at the same rate and held at $50 \%$ FP for rest of the duration. It can be seen from Fig. 6 that the proposed RSPC tracks the demand power variation better than that of the SPC in spite of the uncertainties due to internal reactivity feedback. The time variations of control input and rate of change of control input are shown in Figs. 7 and 8, respectively. The variation of the sliding surface is shown in Fig. 9. Thus, the proposed RSPC controller is found to be robust in the face of uncertainty presented in the input channel.

\section{CONCLuSions}

A robust subspace predictive control approach based on integral sliding mode control is designed to control a PWRtype nuclear reactor. The proposed controller is realized by integrating a nominal control scheme designed using SPC with a discontinuous control scheme designed using ISMC. The proposed RSPC methodology consolidates the advantages of SPC and ISMC and, tries to alleviate their respective drawbacks. The controller is implemented to study two different load-following operations in a PWR.
Simulation results demonstrate that the proposed control strategy maintains the desired performance effectively in the presence of uncertainties. The future work is to generalize the framework to be able to handle unmatched uncertainties.

\section{ACKNOWLEDGEMENT}

The work presented in this paper has been financially supported under grants EP/R021961/1 and EP/R022062/1 from the Engineering and Physical Sciences Research Council.

\section{REFERENCES}

[1] J. B. Rawlings, "Tutorial overview of model predictive control," IEEE Control Systems, vol. 20, no. 3, pp. 38-52, Jun 2000.

[2] H. Eliasi, M. B. Menhaj, and H. Davilu, "Robust nonlinear model predictive control for a PWR nuclear power plant," Progress in Nuclear Energy, vol. 54, pp. 177-185, 2012.

[3] W. Favoreel and B. De Moor, "SPC: Subspace predictive control," in Proc. of the IFAC World Congress, Beijing, 1998, pp. 235-240.

[4] R. Kadali, B. Huang, and A. Rossiter, "A data driven subspace approach to predictive controller design," Control Engineering Practice, vol. 11, no. 3, pp. 261-278, 2003.

[5] V. Vajpayee, S. Mukhopadhyay, and A. P. Tiwari, "Subspace-based wavelet preprocessed data-driven predictive control," INCOSE International Symposium, vol. 26, no. s1, pp. 357-371, 2016.

[6] - "Data-driven subspace predictive control of a nuclear reactor," IEEE Transactions on Nuclear Science, vol. 65, no. 2, pp. 666-679, Feb 2018

[7] A. Bemporad, F. Borrelli, and M. Morari, "Min-max control of constrained uncertain discrete-time linear systems," IEEE Transactions on Automatic Control, vol. 48, no. 9, pp. 1600-1606, Sep. 2003.

[8] L. Chisci, J. Rossiter, and G. Zappa, "Systems with persistent disturbances: predictive control with restricted constraints," Automatica, vol. 37, no. 7, pp. 1019-1028, 2001.

[9] R. C. Shekhar and J. M. Maciejowski, "Optimal constraint tightening policies for robust variable horizon model predictive control," in 2012 IEEE 51st IEEE Conference on Decision and Control (CDC), Dec 2012, pp. 5170-5175.

[10] Y. Liao and J. K. Hedrick, "Robust model predictive control with discrete-time integral sliding surface," in 2015 American Control Conference (ACC), July 2015, pp. 1641-1646.

[11] G. P. Incremona, A. Ferrara, and L. Magni, "MPC for robot manipulators with integral sliding modes generation," IEEE/ASME Transactions on Mechatronics, vol. 22, no. 3, pp. 1299-1307, June 2017.

[12] C. Edwards and S. K. Spurgeon, Sliding Mode Control: Theory and Applications. Taylor and Francis, 1998.

[13] V. Utkin and J. Shi, "Integral sliding mode in systems operating under uncertainty conditions," in Proceedings of 35th IEEE Conference on Decision and Control, vol. 4, Dec 1996, pp. 4591-4596 vol.4.

[14] M. Perez, E. Jimenez, and E. F. Camacho, "Design of an explicit constrained predictive sliding mode controller," IET Control Theory Applications, vol. 4, no. 4, pp. 552-562, April 2010.

[15] K. R. Muske, H. Ashrafiuon, S. Nersesov, and M. Nikkhah, "Optimal sliding mode cascade control for stabilization of underactuated nonlinear systems," Journal of Dynamic Systems, Measurement, and Control, vol. 134, no. 2, jan 2012.

[16] A. Musa, L. R. Sabug, and A. Monti, "Robust predictive sliding mode control for multiterminal HVDC grids," IEEE Transactions on Power Delivery, vol. 33, no. 4, pp. 1545-1555, Aug 2018.

[17] M. Rubagotti, D. M. Raimondo, A. Ferrara, and L. Magni, "Robust model predictive control with integral sliding mode in continuous-time sampled-data nonlinear systems," IEEE Transactions on Automatic Control, vol. 56, no. 3, pp. 556-570, March 2011.

[18] G. P. Incremona, A. Ferrara, and L. Magni, "Asynchronous networked MPC with ISM for uncertain nonlinear systems," IEEE Transactions on Automatic Control, vol. 62, no. 9, pp. 4305-4317, Sep 2017.

[19] R. Errouissi, J. Yang, W. Chen, and A. Al-Durra, "Robust nonlinear generalised predictive control for a class of uncertain nonlinear systems via an integral sliding mode approach," International Journal of Control, vol. 89, no. 8, pp. 1698-1710, 2017.

[20] P. V. Surjagade, A. P. Tiwari, and S. R. Shimjith, "Robust optimal integral sliding mode controller for total power control of large PHWRs," IEEE Transactions on Nuclear Science, vol. 65, no. 7, pp. 1331-1344, July 2018. 